\title{
Fatores facilitadores e barreiras ao trabalho em equipe para atendimento hospitalar de acidente vascular cerebral
}

\author{
Facilitating factors and barriers to teamwork for hospital care for stroke \\ Factores facilitadores y barreras al trabajo en equipo para la atención hospitalaria por accidente
} cerebrovascular

\author{
Daniela Carrogi-Vianna \\ ORCID: https://orcid.org/0000-0003-3897-4727 \\ Universidade Estadual de Campinas, Brasil \\ E-mail: d107220@dac.unicamp.br \\ Ana Railka de Souza Oliveira-Kumakura \\ ORCID: https://orcid.org/0000-0002-7075-7987 \\ Universidade Estadual de Campinas, Brasil \\ E-mail: arailka@unicamp.br \\ José Luiz Tatagiba Lamas \\ ORCID: https://orcid.org/0000-0003-4266-6209 \\ Universidade Estadual de Campinas, Brasil \\ E-mail: zelamas@unicamp.br
}

\begin{abstract}
Resumo
Objetivos: Identificar fatores facilitadores e barreiras ao trabalho realizado por equipes multidisciplinares e interdisciplinares nos pacientes com AVC em hospitais e identificar os profissionais de saúde envolvidos. Método: Realizou-se uma revisão integrativa com busca nas bases: Biblioteca Virtual em Saúde, CINAHL, Cochrane, Embase, PEDro, ProQuest, PubMed, Scopus e Web of Science. Foram incluídos estudos primários publicados entre 2014 e 2020, em inglês, português e espanhol, disponíveis na íntegra e que responderam à questão norteadora da revisão. Resultados: Foram selecionados oito estudos primários, cujos resultados mostraram uma diversidade de trabalhos desenvolvidos pelas equipes, por conta da grande demanda de cuidados necessários para a população com AVC. Houve grande variação de profissionais nas equipes, sendo que apenas enfermeiros estiveram presentes em todas. Foram identificados barreiras e facilitadores ao trabalho em equipe, relacionados às necessidades específicas desses pacientes. Como facilitadores apareceram a colaboração e a comunicação aprimorada. Como barreiras foram encontradas falta de tempo e falta de envolvimento de membros da equipe. Conclusões: É importante entender como os diferentes profissionais das equipes atuam juntos no trabalho com pacientes de AVC, pois, isso é crucial para avaliar integralmente o contexto dessas pessoas com essa doença crônica e planejar o cuidado, de maneira holística.
\end{abstract}

Palavras-chave: Acidente Vascular Cerebral (AVC); Equipe interdisciplinar de saúde; Equipe de assistência médica; Equipes de assistência ao paciente; Hospital.

\begin{abstract}
Objectives: To identify facilitators and barriers to the work carried out by multidisciplinary and interdisciplinary teams to stroke patients in hospitals and to identify the health professionals involved. Method: An integrative review was carried out with the search for bases: Virtual Health Library, CINAHL, Cochrane, Embase, PEDro, ProQuest, PubMed, Scopus and Web of Science. Including primary studies published between 2014 and 2020, in English, Portuguese and Spanish, you have a complete report that will answer the guiding question of the review. Results: Selected primary study forums, whose results show a diversity of work carried out by the teams, due to the high demand for care needed by the population with stroke. There is a wide range of professionals in the teams, with only nurses present in all of them. Barriers and facilitators for or teamwork were identified, related to the specific needs of patients. Collaboration and communication appeared as facilitators. The barriers found were insufficient time and lack of involvement of team members. Conclusions: It is important to understand how the different professionals of the teams work together with stroke patients, as it is so crucial to comprehensively assess the context of their personalities and plan chronic care, in a holistic way.
\end{abstract}

Keywords: Stroke; Interdisciplinary health team; Medical care team; Patient care teams; Hospital.

\section{Resumen}

Objetivos: Identificar factores facilitadores y barreras al trabajo de los equipos multidisciplinares e interdisciplinares en pacientes con accidente cerebrovascular en hospitales e identificar a los profesionales sanitarios involucrados. Método: Se realizó una revisión integradora con búsqueda en las siguientes bases de datos: Virtual Health Library, CINAHL, Cochrane, Embase, PEDro, ProQuest, PubMed, Scopus y Web of Science. Se incluyeron los estudios primarios publicados entre 2014 y 2020, en inglés, portugués y español, disponibles en su totalidad y que respondieron a la pregunta orientadora de la revisión. 
Resultados: Se seleccionaron ocho estudios primarios, cuyos resultados evidenciaron una diversidad de trabajos desarrollados por los equipos, debido a la gran demanda de cuidados que necesita la población con accidente cerebrovascular. Hubo una amplia variación de profesionales en los equipos, con solo enfermeras presentes en todos ellos. Se identificaron barreras y facilitadores para el trabajo en equipo, relacionados con las necesidades específicas de estos pacientes. Como facilitadores surgieron la colaboración y la mejora de la comunicación. Como barreras se encontraron falta de tiempo y falta de participación de los miembros del equipo. Conclusiones: Es importante comprender como los distintos profesionales de los equipos trabajan juntos cuando se trabaja con pacientes con accidente cerebrovascular, ya que esto es crucial para evaluar completamente el contexto de estas personas con esta enfermedad crónica y planear la atención de manera integral.

Palavras clave: Accidente Cerebrovascular; Equipo de salud interdisciplinario; Equipo de asistencia médica; Equipos de atención al paciente; Hospital.

\section{Introdução}

O Acidente Vascular Cerebral (AVC) é definido pela Organização Mundial de Saúde (OMS) como o desenvolvimento rápido de sinais clínicos de distúrbios focais ou globais da função cerebral (Coupland et al., 2017). O número de sobreviventes avança com rapidez, os 33 milhões atuais devem se tornar 77 milhões até 2030 (Feigin, Norrving \& Mensah, 2017; Feigin et al., 2019). Nesse contexto, salienta-se que a doença provoca sequelas, alto risco de hospitalização, grande impacto socioeconômico (Feigin, Norrving \& Mensah, 2017; Koton \& Rexrode, 2017) e a frequência de readmissão após qualquer episódio, pode chegar a 62\% em 1 ano (Bjerkreim et al., 2016).

O AVC é visto como uma emergência médica com vias claras de atendimento baseadas em evidências, desde a admissão até a alta hospitalar, a qual demanda cuidados específicos para o tratamento (Xianmei et. al., 2019). De acordo com a American Heart Association, a previsão dos custos médicos anuais relacionados diretamente ao AVC é de um aumento de U\$ 71,55 bilhões em 2012, para U\$ 183,13 bilhões em 2030 (Philip, 2019).

Frente a isso, há tempos tem sido demonstrado que para a obtenção de uma reabilitação bem sucedida, vários profissionais de saúde com diferentes especialidades clínicas devem trabalhar desde o início do atendimento em equipe, de forma harmoniosa e eficaz e que esse trabalho deveria resultar no alcance dos objetivos de reabilitação para os pacientes. Cada profissional de uma equipe de saúde possui uma habilidade específica para melhorar o atendimento. A combinação dessas habilidades permite o acesso a uma gama maior de conhecimentos sobre a avaliação e diagnóstico de uma condição, a mensuração de restrições e limitações de uma atividade que levará ao estabelecimento de metas e a seleção de opções de tratamento adequadas. Por conseguinte, a medida dos resultados deve ser documentada para mostrar a eficácia do serviço (Singh et al., 2018).

Nesse contexto, os profissionais envolvidos no atendimento a determinada alteração da saúde frequentemente se organizam em equipes multidisciplinares (EMD's), equipes interdisciplinares (EID's) ou equipes transdisciplinares (ETD's). Uma EMD, composta por diferentes profissionais que compartilham um trabalho comum, realiza eficazmente o trabalho de disciplinas individuais, mas os benefícios da ação em equipe podem não ser percebidos (Becker et al., 2017; Singh et al., 2018). Já, o trabalho das EID's implica que os membros da equipe aceitam, além do trabalho comum, a responsabilidade adicional do esforço do grupo em nome dos pacientes (Singh et al., 2018), além de contribuírem com diferentes perspectivas profissionais, estabelecerem metas, planejarem cuidados e tomarem decisões em grupo (Royal College Of Physicians, 2017; Ranford et al., 2019; Walton et al., 2019). Em uma ETD, os limites da prática profissional confundem-se, pois, qualquer profissional é capaz de trabalhar em qualquer função específica da equipe, o que gera mais flexibilidade no tratamento, no entanto, isso exige uma equipe treinada em uma série de habilidades ou profissões, o que consome tempo e recursos à medida que os membros da equipe mudam. Sendo assim, por conta de todos esses fatores, a EMD e a EID tem sido mais utilizadas em cuidados hospitalares e também na reabilitação (Singh, et al., 2018).

Diversas condições patológicas são tratadas por EMD ou EID. Há relatos de atuação em equipe na abordagem a doenças crônicas, como o AVC, foco deste estudo (Xianmei, et al., 2019; Ngangue, et al., 2020). Os atendimentos 
ambulatoriais organizados em unidades de AVC por EMD e EID melhoram os resultados e as vantagens obtidas são persistentes, o que os torna um princípio central dos cuidados de alta qualidade para o AVC (Hustoft et al., 2018), que possui como principais causas de readmissão, as infecções, as doenças cerebrovasculares e as cardiovasculares (Philip, 2019). Porém, nem todos os serviços dispõem de profissionais suficientes para formar essas equipes, o que levanta a possibilidade de heterogeneidade em sua constituição (Hustoft et al., 2018).

Logo, o objetivo desta pesquisa é identificar fatores facilitadores e barreiras ao trabalho realizado por equipes multidisciplinares (EMD’s) e interdisciplinares (EID’s) nos pacientes com AVC em hospitais e identificar quais profissionais de saúde estão envolvidos.

\section{Metodologia}

Trata-se de uma revisão integrativa (RI), método adequado para analisar e sintetizar pesquisas de forma sistematizada, contribuir para a tomada de decisão, aprofundar o tema e melhorar a prática clínica (Mendes, Silveira \& Galvão, 2008). Nesta RI, os seguintes passos foram seguidos: 1. Identificação do tema e elaboração da questão de pesquisa; 2. Estabelecimento de critérios de inclusão e exclusão; 3. Coleta de dados ou definições sobre a busca na literatura; 4. Avaliação do nível de evidência; 5. Interpretação e síntese dos resultados (Mendes, Silveira \& Galvão, 2008).

A questão norteadora (passo 1) foi: "Como funciona o trabalho das equipes multidisciplinares e interdisciplinares envolvidas no cuidado hospitalar a pacientes diagnosticados com AVC e quais são os profissionais de saúde que as integram?"

Os critérios de inclusão (passo 2) foram: estudos primários e originais, publicados entre 2014 e 2020, em português, inglês e espanhol e disponíveis eletronicamente, na íntegra. Os critérios de exclusão foram: estudos de revisão, resumos de congressos, editoriais, artigos em formato de diretrizes, relatos de experiência, teses, dissertações e artigos originais com participantes menores de 18 anos.

A coleta dos dados ou busca da literatura (passo 3), foi realizada no período de Julho a Setembro de 2020. Os artigos foram selecionados por dois revisores e discordâncias entre eles foram resolvidas por um terceiro. As bases de dados pesquisadas, foram: Biblioteca Virtual em Saúde (BVS), Cumulative Index to Nursing and Allied Health Literature (CINAHL), Colaboração COCHRANE, Base de dados internacional de ciências biomédicas (EMBASE), Base de dados de evidências em fisioterapia (PEDRO), Base de dados da área multidisciplinar (PROQUEST), National Library of Medicine National Institutes of Health (PubMed), Base de dados da área multidisciplinar (SCOPUS) e Base de dados da área multidisciplinar (Web of Science). Essas bases foram acessadas por meio de permissão de acesso remoto pela Universidade Estadual de Campinas (UNICAMP).

Foram identificados 5826 artigos, sendo incluídos 8 nessa RI. O detalhamento da seleção dos artigos é apresentado na Figura 1, de acordo com a recomendação PRISMA (Mother et al., 2009). Destaca-se que 16 artigos foram excluídos após a ação da terceira revisora, que considerou tratarem de outra temática. 
Figura 1 - Descrição do processo de seleção dos artigos de acordo com o PRISMA (Mother et al., 2009).

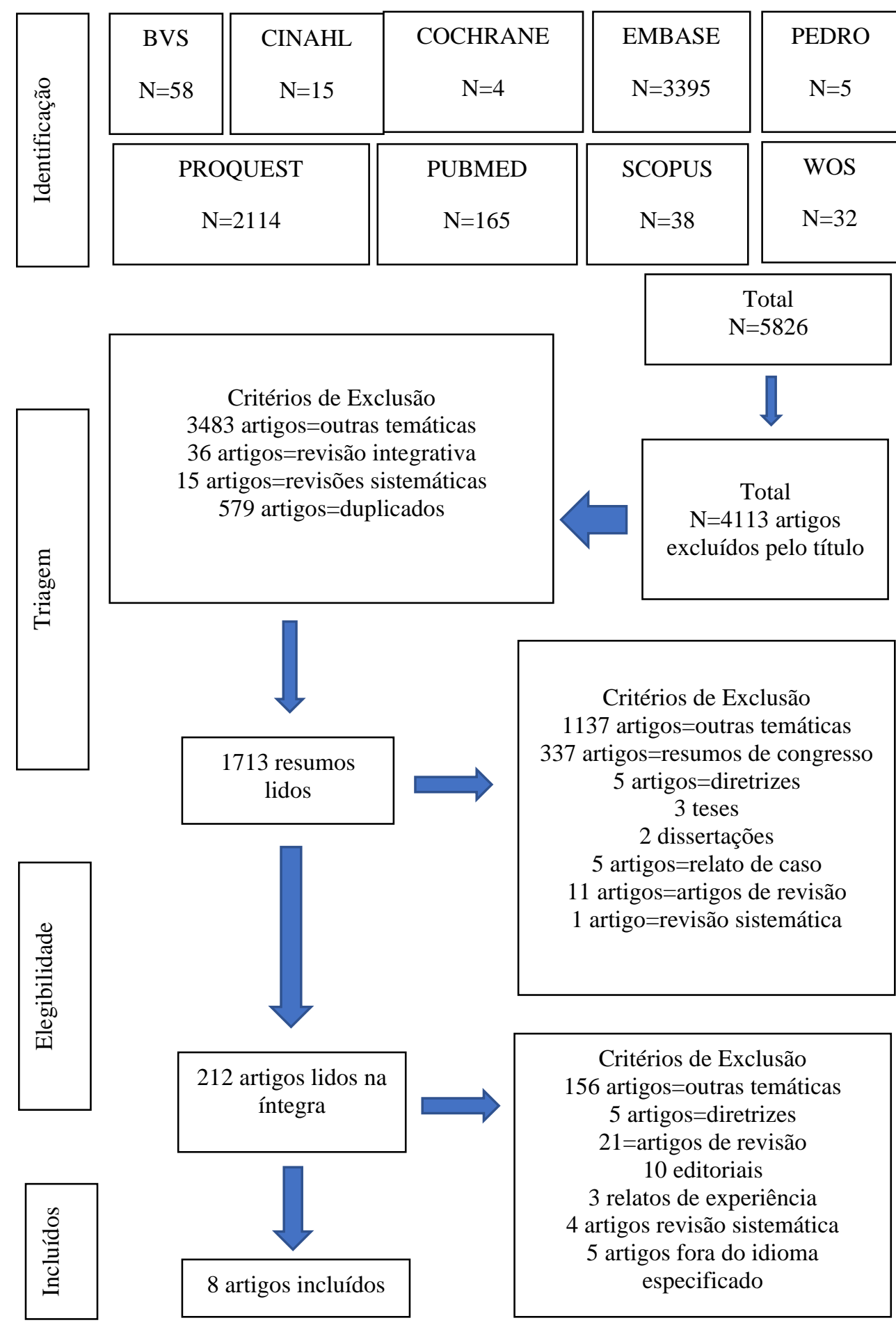

Fonte: Autores.

Os artigos foram obtidos utilizando descritores do vocabulário controlado específico de cada base, relacionados ao AVC, trabalho em equipe, hospital, além dos nomes dos profissionais e das profissões envolvidas (ex.: fisioterapia e fisioterapeuta) na EMD da instituição de origem dos autores. A busca foi realizada exclusivamente com termos em inglês e orientada por uma bibliotecária.

Na primeira estratégia, os descritores ou seus sinônimos foram combinados com o operador boleano "OU". Quando não havia o descritor específico na base, era utilizada uma palavra-chave que se aproximasse da definição desejada. Para a 
segunda estratégia, os descritores foram combinados utilizando o operador booleano "E", até obter estudos que atendessem aos critérios de inclusão e exclusão.

$\mathrm{Na}$ etapa de coleta de dados foi realizada a leitura dos artigos que atenderam aos critérios de inclusão na íntegra. Para extração dos dados foi utilizado um instrumento, construído pelos autores, contendo informações relacionadas ao estudo, como: referência, número da referência, equipe de saúde, objetivo, modelo de trabalho utilizado, resultados do trabalho em equipe, facilitadores e barreiras (Ursi \& Galvão, 2006). Os resultados foram sintetizados destacando-se as informações chave de cada artigo. Os estudos receberam código de sequência numérica, para facilitar a identificação e apresentação dos resultados, por exemplo: Estudo 1(E1), 2(E2), 3(E3), assim por diante e foram classificados de acordo com o nível de evidência (passo 4). Quanto a esse último quesito, considerou-se a seguinte hierarquia: I - Revisão Sistemática ou metaanálise; II - Teste controlado e aleatório; III - Ensaio controlado sem randomização; IV - Estudo de caso-controle ou coorte; V - Revisão Sistemática de estudos qualitativos ou descritivos; VI - Estudo qualitativo ou descritivo; VII - Opiniões ou consensos (Stillwel et al., 2010).

A análise dos dados (passo 5) foi feita mediante leitura dos artigos na íntegra, síntese do conteúdo e preenchimento do instrumento supramencionado, sendo possível identificar diferentes aspectos que influenciavam o tipo de trabalho das equipes interdisciplinares e multidisciplinares. Esses aspectos foram analisados em detalhes e agrupados de forma que facilitasse a integração entre os achados, revelando-se dessa observação, duas categorias:

1. "Como funciona o trabalho interdisciplinar e multidisciplinar no atendimento de AVC em hospitais e quais fatores influenciam os resultados do trabalho em equipe" e 2. "Quais os profissionais envolvidos nas equipes"

$\mathrm{Na}$ etapa de apresentação e interpretação, foi adotada a forma descritiva para exposição dos resultados em um quadro, sendo apresentada a síntese de cada estudo e as comparações entre as pesquisas e suas limitações.

\section{Resultados}

O Quadro 1 resume as características dos estudos incluídos na revisão. Três estudos foram desenvolvidos no Canadá: E1(Johnson et al., 2015), E2 (Singh et al., 2015) e E4 (Munce et al., 2017); dois nos Estados Unidos da América: E6 (Abhijit et al., 2019) e E8 (Yang et al., 2019); um na Itália: E3 (Liberati et al., 2016); um no Japão: E7 (Obana et al., 2019) e um no Reino Unido: E5 (Plant \& Tyson, 2018), sendo todos publicados em inglês. Todos os autores eram vinculados a universidades. Duas pesquisas foram publicadas em 2015, uma em 2016, uma em 2017, uma em 2018 e três em 2019.

Em relação ao tipo de artigo, foram identificados 6 estudos descritivos qualitativos e 2 estudos etnográficos qualitativos. Quanto ao nível de evidência, todos os artigos foram classificados como nível VI. 
Quadro 1 - Síntese dos resultados encontrados em cada artigo, apresentados em ordem cronológica.

\begin{tabular}{|c|c|c|c|c|c|c|}
\hline Referência & $\begin{array}{l}\text { Equipe de } \\
\text { Saúde }\end{array}$ & Objetivo & $\begin{array}{c}\text { Modelo de } \\
\text { Trabalho } \\
\text { Utilizado }\end{array}$ & $\begin{array}{c}\text { Resultados do } \\
\text { Trabalho em Equipe }\end{array}$ & Facilitadores & Barreiras \\
\hline $\begin{array}{l}\text { E2 (Singh et } \\
\text { al., 2015) }\end{array}$ & $\begin{array}{l}\text { Enfermeir } \\
\text { o, } \\
\text { Fisioterap } \\
\text { euta, } \\
\text { Neurologi } \\
\text { sta e } \\
\text { Terapeuta } \\
\text { Ocupacio } \\
\text { nal. }\end{array}$ & $\begin{array}{lr}\text { Avaliar } & \text { e } \\
\text { determinar } & \mathrm{a} \\
\text { eficácia } & \text { da } \\
\text { utilização de } & \text { uma } \\
\text { equipe } & \text { de } \\
\text { transferência } & \text { de } \\
\text { conhecimento } & \\
\text { (ETC) } & \\
\text { para implementar } \\
\text { e disseminar } \\
\text { diretrizes r de } \\
\text { melhores práticas } \\
\text { (DMP) rriadas } \\
\text { pela associação de } \\
\text { enfermeiros r de } \\
\text { Ontário r para } \\
\text { avaliação } & \text { do } \\
\text { AVC. } & \end{array}$ & $\begin{array}{lr}\text { Discussão } & \text { das } \\
\text { recomendações } & \text { das } \\
\text { DMP } & \text { e } \\
\text { compartilhamento } \\
\text { de dicas, desafios e } \\
\text { ensinamentos } & \text { sobre } \\
\text { habilidades } & \\
\text { necessárias r para } \\
\text { concluir } \\
\text { avaliações de } & \text { AVC. }\end{array}$ & $\begin{array}{l}\text { A abordagem da ETC } \\
\text { resultou em melhor } \\
\text { atendimento ao paciente, } \\
\text { demonstrado pelos altos } \\
\text { níveis de satisfação dos } \\
\text { atendidos. }\end{array}$ & $\begin{array}{l}\text { Colaboração e } \\
\text { comunicação } \\
\text { aprimorada da } \\
\text { equipe. }\end{array}$ & $\begin{array}{l}\text { Amostra } \\
\text { pequena de } \\
\text { dados dos } \\
\text { pacientes e da } \\
\text { equipe do } \\
\text { hospital, } \\
\text { prioridades } \\
\text { conflitantes e } \\
\text { atritos entre } \\
\text { membros da } \\
\text { equipe, tempo } \\
\text { reduzido e falta } \\
\text { de apoio } \\
\text { financeiro para } \\
\text { o trabalho } \\
\text { adicional da } \\
\text { equipe com a } \\
\text { implementação } \\
\text { da DMP. }\end{array}$ \\
\hline $\begin{array}{l}\text { E3 (Liberati } \\
\text { et al., 2016) }\end{array}$ & $\begin{array}{l}\text { Enfermeir } \\
\text { o e } \\
\text { Neurologi } \\
\text { sta. }\end{array}$ & $\begin{array}{l}\text { Analisar: 1. Como } \\
\text { ocorre o trabalho } \\
\text { por meio das } \\
\text { práticas cotidianas } \\
\text { de cuidado em } \\
\text { equipes } \\
\text { multidisciplinares } \\
\text { (EMD's) recém } \\
\text { criadas (de cima } \\
\text { para baixo); } 2 \text { se e } \\
\text { como os limites } \\
\text { disciplinares } \\
\text { afetam o trabalho } \\
\text { de médicos e } \\
\text { enfermeiras; } 3 \text {. Se } \\
\text { e como os limites } \\
\text { disciplinares }\end{array}$ & $\begin{array}{l}\text { Médicos de } \\
\text { diferentes } \\
\text { especialidades usam } \\
\text { abordagens } \\
\text { diferentes, às vezes } \\
\text { conflitantes. } \\
\text { Médicos } \\
\text { enfermeiros } \\
\text { constroem } \\
\text { identidades } \\
\text { profissionais } \\
\text { específicas. }\end{array}$ & $\begin{array}{lrr}\text { Um } & \text { trabalho } \\
\text { interdisciplinar } & \text { exige a } \\
\text { ligação entre } & \text { fronteiras } \\
\text { intraprofissionais } & \text { e } & \text { a } \\
\text { consideração da dinâmica } & \\
\text { do controle } & \text { e } & \text { da } \\
\text { resistência dos envolvidos } \\
\text { na fusão de } & \text { médicos e } \\
\text { enfermeiros } & \text { com } \\
\text { diferentes especialidades. }\end{array}$ & $\begin{array}{l}\text { Reuniões } \\
\text { regulares das } \\
\text { EMD's } \\
\text { poderiam ser } \\
\text { mediadas por } \\
\text { um facilitador, } \\
\text { pesquisador } \\
\text { ou consultor } \\
\text { familiarizado } \\
\text { com o } \\
\text { contexto local, } \\
\text { a fim de } \\
\text { produzir } \\
\text { novas interpretações } \\
\text { compartilhada } \\
\text { s que possam }\end{array}$ & $\begin{array}{l}\text { Há conflitos } \\
\text { entre médicos e } \\
\text { enfermeiros de } \\
\text { diferentes } \\
\text { especialidades } \\
\text { sobre a } \\
\text { regulação da } \\
\text { fronteira } \\
\text { médico- } \\
\text { enfermagem. }\end{array}$ \\
\hline
\end{tabular}




\begin{tabular}{|c|c|c|c|c|c|c|}
\hline & & $\begin{array}{l}\text { afetam a prestação } \\
\text { de cuidados } \\
\text { integrados ao } \\
\text { paciente. }\end{array}$ & & & $\begin{array}{ll}\text { informar } & \text { as } \\
\text { práticas } & \text { de } \\
\text { cuidado } & \\
\text { futuras. } & \end{array}$ & \\
\hline $\begin{array}{l}\text { E4 (Munce } \\
\text { et al.,2017) }\end{array}$ & $\begin{array}{l}\text { Enfermeir } \\
\text { o, } \\
\text { Fisioterap } \\
\text { euta e } \\
\text { Terapeuta } \\
\text { Ocupacio } \\
\text { nal. }\end{array}$ & 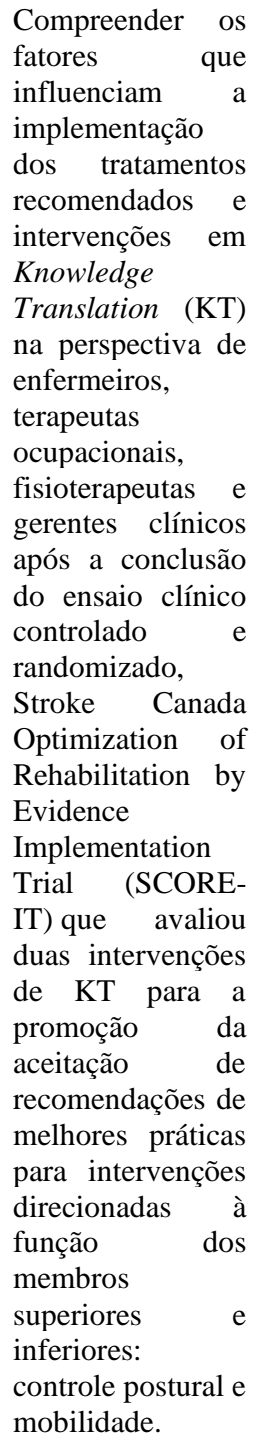 & $\begin{array}{l}\text { Intervenções em } \\
\text { Knowledge } \\
\text { Translation (KT). }\end{array}$ & $\begin{array}{l}\text { O trabalho em equipe } \\
\text { pode ter uma influência } \\
\text { significativa não apenas } \\
\text { na melhoria da } \\
\text { implementação das } \\
\text { diretrizes, mas também } \\
\text { nos ganhos funcionais } \\
\text { do paciente e no tempo } \\
\text { de internação hospitalar. }\end{array}$ & $\begin{array}{l}\text { Facilitação } \\
\text { (permitindo } \\
\text { que } \\
\text { indivíduos, } \\
\text { equipes e } \\
\text { organizações } \\
\text { mudem), } \\
\text { concordância } \\
\text { com a } \\
\text { intervenção/pr } \\
\text { ática, } \\
\text { familiaridade } \\
\text { com os } \\
\text { tratamentos } \\
\text { recomendados } \\
\text { ambientais e } \\
\text { principalment } \\
\text { e a } \\
\text { comunicação } \\
\text { aprimorada da } \\
\text { equipe e } \\
\text { colaboração } \\
\text { interdisciplina } \\
\text { r. }\end{array}$ & $\begin{array}{l}\text { Falta de espaço } \\
\text { e equipamento } \\
\text { e restrições } \\
\text { organizacionais } \\
\text {. }\end{array}$ \\
\hline $\begin{array}{l}\text { E5 (Plant \& } \\
\text { Tyson, 2018) }\end{array}$ & $\begin{array}{l}\text { Assistente } \\
\text { Social, } \\
\text { Enfermeir } \\
\text { o, } \\
\text { Fisioterap } \\
\text { euta, } \\
\text { Fonoaudió } \\
\text { logo, } \\
\text { Neurologi } \\
\text { sta, } \\
\text { Nutricioni } \\
\text { sta e } \\
\text { Terapeuta } \\
\text { Ocupacio } \\
\text { nal. }\end{array}$ & 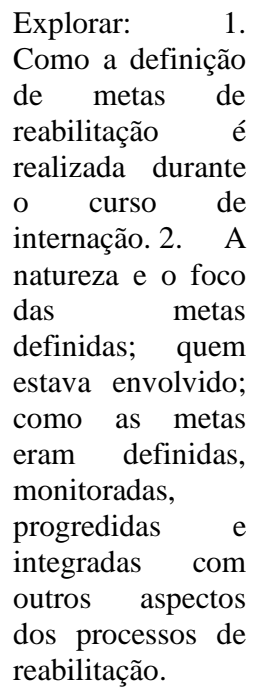 & $\begin{array}{l}\text { Estabelecimento de } \\
\text { metas em reuniões } \\
\text { semanais, realização } \\
\text { de avaliações, } \\
\text { acompanhamento da } \\
\text { evolução e planos } \\
\text { de ação/ tratamento, } \\
\text { revisão do } \\
\text { progresso e registro } \\
\text { da revisão e do } \\
\text { alcance ou não da } \\
\text { meta. } \\
\text { Posteriormente, } \\
\text { planos de ação e } \\
\text { tratamento surgiram } \\
\text { da reunião da } \\
\text { equipe. }\end{array}$ & $\begin{array}{l}\text { O foco das metas foi } \\
\text { predominantemente } \\
\text { relacionado ao paciente } \\
\text { quanto a suas } \\
\text { deficiências (mobilidade } \\
\text { e transferências), } \\
\text { seguido da equipe } \\
\text { multidisciplinar e da } \\
\text { família, como último } \\
\text { item. } \\
\text { Em todas as unidades, os } \\
\text { problemas e metas foram } \\
\text { identificados e definidos } \\
\text { pela equipe: } 109 \text { (31\%) } \\
\text { por um terapeuta } \\
\text { ocupacional, 95 (27\%) } \\
\text { por um fisioterapeuta e } 7 \\
\text { (2\%) por um } \\
\text { fonoaudiólogo. }\end{array}$ & $\begin{array}{l}\text { Terapeutas } \\
\text { identificando } \\
\text { a maior parte } \\
\text { de problemas } \\
\text { e objetivos } \\
\text { relacionados } \\
\text { às deficiências } \\
\text { dos pacientes } \\
\text { com AVC. }\end{array}$ & $\begin{array}{l}\text { Falta de tempo } \\
\text { e de } \\
\text { envolvimento } \\
\text { de alguns } \\
\text { membros da } \\
\text { equipe } \\
\text { limitações } \\
\text { relacionadas ao } \\
\text { AVC } \\
\text { paciente. }\end{array}$ \\
\hline
\end{tabular}




\begin{tabular}{|c|c|c|c|c|c|c|}
\hline $\begin{array}{l}\text { E6 (Abhijit } \\
\text { et al., 2019) }\end{array}$ & $\begin{array}{l}\text { Médico } \\
\text { Intensivist } \\
\text { a, } \\
\text { Médicos } \\
\text { Residente } \\
\text { s em } \\
\text { Anestesiol } \\
\text { ogia e } \\
\text { Enfermeir } \\
\text { as. }\end{array}$ & 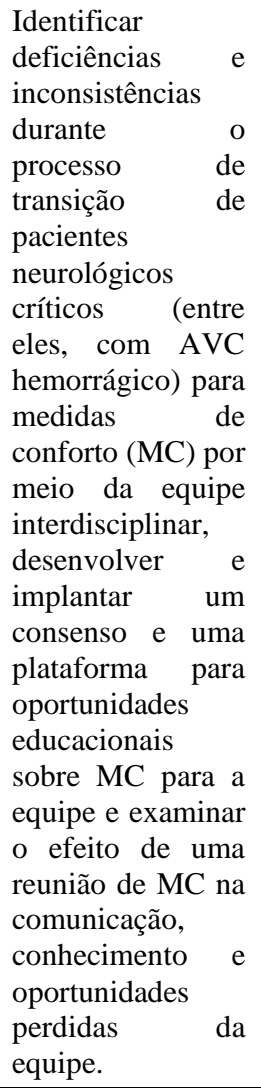 & $\begin{array}{l}\text { Reuniões semanais } \\
\text { para a realização de } \\
\text { discussões sobre } \\
\text { MC. }\end{array}$ & $\begin{array}{l}64,3 \% \text { dos enfermeiros } \\
\text { relataram que se } \\
\text { sentiram mais incluídos } \\
\text { e envolvidos nos } \\
\text { processos de MC, } \\
\text { comparado a } 28 \% \text { antes } \\
\text { da implementação da } \\
\text { MC, } 87,9 \% \text { dos } \\
\text { médicos intensivistas } \\
\text { relataram se sentir } \\
\text { confortáveis } \\
\text { participando } \\
\text { discussões de MC, em } \\
\text { comparação com } 69,8 \% \\
\text { antes, } 57 \% \text { dos médicos } \\
\text { relataram que a } \\
\text { comunicação entre eles } \\
\text { também melhorou, } \\
51,9 \% \text { relataram redução } \\
\text { das chances perdidas } \\
\text { durante o processo de } \\
\text { MC e } 21,7 \% \text { relataram } \\
\text { que o processo atual de } \\
\text { MC é inferior ao ideal, } \\
\text { em comparação com } \\
80 \% \text { antes. }\end{array}$ & $\begin{array}{l}\text { Colaboração e } \\
\text { comunicação } \\
\text { aprimorada. }\end{array}$ & $\begin{array}{l}\text { Falta de } \\
\text { consenso } \\
\text { multidisciplinar } \\
\text { na unidade de } \\
\text { terapia } \\
\text { intensiva antes } \\
\text { da transição } \\
\text { para MC. }\end{array}$ \\
\hline $\begin{array}{l}\text { E7 (Obana et } \\
\text { al., 2019) }\end{array}$ & $\begin{array}{l}\text { Dentistas, } \\
\text { Enfermeir } \\
\text { as, } \\
\text { Fonoaudió } \\
\text { logos e } \\
\text { Higienista } \\
\text { s Orais. }\end{array}$ & $\begin{array}{l}\text { Objetivou-se } \\
\text { identificar o } \\
\text { estado de saúde } \\
\text { bucal de pacientes } \\
\text { com AVC na fase } \\
\text { aguda usando o } \\
\text { OHAT (Oral } \\
\text { Health } \\
\text { Assessment Tool) } \\
\text { e elucidar os } \\
\text { efeitos de um } \\
\text { cuidado } \\
\text { colaborativo de } \\
\text { saúde bucal com } \\
\text { uma abordagem } \\
\text { de equipe } \\
\text { transdisciplinar. }\end{array}$ & 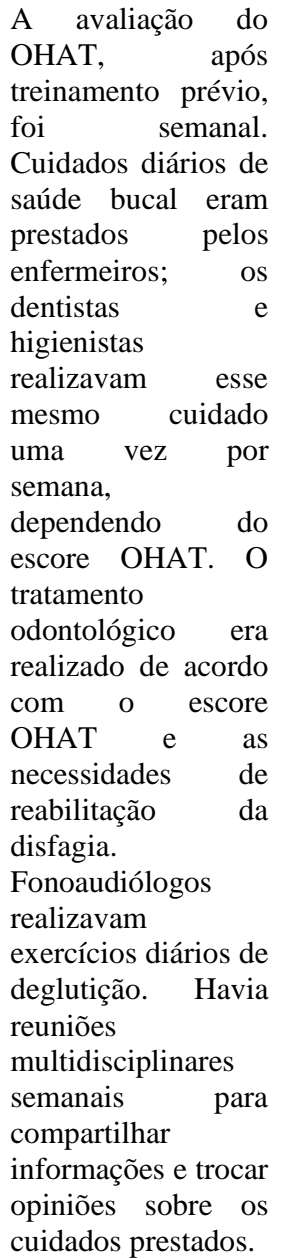 & $\begin{array}{lr}\text { Diminuição significativa } \\
\text { dos escores } & \text { OHAT na } \\
\text { alta. A r análise } \\
\text { multivariada identificou } \\
\text { a pontuação OHAT para } \\
\text { língua, dentaduras e } \\
\text { limpeza oral na } \\
\text { admissão como as } \\
\text { variáveis significativas } \\
\text { associadas ao mau } \\
\text { estado de saúde bucal na } \\
\text { alta. }\end{array}$ & $\begin{array}{l}\text { Colaboração e } \\
\text { comunicação } \\
\text { aprimorada. }\end{array}$ & $\begin{array}{l}\text { Não } \\
\text { mencionado. }\end{array}$ \\
\hline
\end{tabular}




\begin{tabular}{|c|c|c|c|c|c|c|}
\hline $\begin{array}{l}\text { E8 (Yang et } \\
\text { al., 2019) }\end{array}$ & $\begin{array}{l}\text { Neurologi } \\
\text { sta, } \\
\text { Radiologi } \\
\text { sta, } \\
\text { Enfermeir } \\
\text { o e } \\
\text { Farmacêut } \\
\text { ico. }\end{array}$ & $\begin{array}{l}\text { Observar a } \\
\text { eficácia de um } \\
\text { protocolo } \\
\text { interdisciplinar de } \\
\text { código de AVC } \\
\text { (Code Stroke: } \\
\text { profissionais } \\
\text { sendo recrutados } \\
\text { ao mesmo tempo } \\
\text { para realizar } \\
\text { intervenção nos } \\
\text { pacientes com } \\
\text { AVC) dirigido por } \\
\text { enfermeiras para } \\
\text { reconhecimento e } \\
\text { tratamento de } \\
\text { AVC isquêmico } \\
\text { em pacientes } \\
\text { internados. }\end{array}$ & 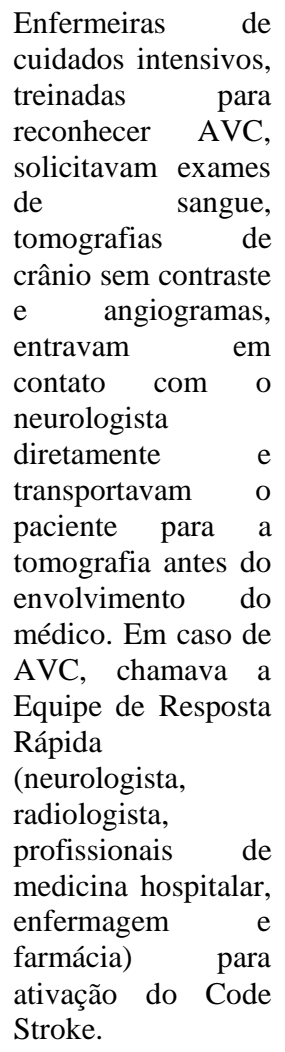 & $\begin{array}{l}\text { Houve redução } \\
\text { significativa no tempo } \\
\text { de reconhecimento do } \\
\text { início dos sintomas de } \\
\text { AVC até a obtenção de } \\
\text { imagens cerebrais (de } 69 \\
\text { para } 37 \text { minutos; p= } \\
0,002) \text {. }\end{array}$ & $\begin{array}{l}\text { Envolvimento } \\
\text { da } \\
\text { enfermagem } \\
\text { na ativação do } \\
\text { Code Stroke. }\end{array}$ & $\begin{array}{l}\text { Não } \\
\text { mencionado. }\end{array}$ \\
\hline
\end{tabular}

Fonte: Autores.

Categoria 1. "Como funciona o trabalho interdisciplinar e multidisciplinar no atendimento de AVC em hospitais e quais fatores influenciam os resultados do trabalho em equipe"

Com base nesses resultados, constatou-se que cinco estudos abordaram como a colaboração e a comunicação aprimorada no trabalho das equipes interdisciplinares e multidisciplinares pode funcionar como um facilitador para a tomada de decisões em intervenções nos pacientes com AVC e em cinco diferentes quesitos, sendo que cada um deles é citado a seguir: trabalho da equipe interdisciplinar sobre a alta hospitalar (E1) (Johnson et al., 2015), sobre a avaliação da utilização de uma equipe interdisciplinar de transferência do conhecimento (ETC) para implementar e disseminar recomendações de diretrizes de melhores práticas para avaliação do AVC (E2) (Singh et al., 2015), compreensão dos fatores que influenciam a implementação dos tratamentos recomendados e intervenções em Knowledge Translation (KT) na perspectiva da equipe interdisciplinar (E4) (Munce et al., 2017), sobre o aprimoramento da comunicação como resultado da implementação de consenso interdisciplinar de medidas de conforto para o paciente com AVC (E6) (Abhijit et al., 2019) e acerca da realização de cuidados colaborativos da equipe transdisciplinar relacionados à saúde bucal desses pacientes (E7) (Obana et al., 2019).

Constatou-se também, em quatro estudos, que houve abordagem de temas diferentes nos resultados, mas todos relacionados ao trabalho de equipes interdisciplinares e multidisciplinares de AVC, como: trabalho da equipe multidisciplinar sob a regulação da fronteira médico-enfermagem (E3) (Liberati et al., 2016), maior parte da identificação de problemas e objetivos relacionados às deficiências dos pacientes com AVC, sendo realizada pelos terapeutas da equipe multidisciplinar (E5) (Plant \& Tyson, 2018), implementação de consenso interdisciplinar de medidas de conforto para o paciente com AVC, facilitando a inclusão de enfermeiros e proporcionando maior sensação de comodidade aos médicos para sua participação nas reuniões sobre esse processo (E6) (Abhijit et al., 2019) e melhora no tempo de reconhecimento do início dos sintomas de ocorrência de AVC em pacientes internados, por meio da equipe interdisciplinar, dirigido por enfermeiras (E8) (Yang et al., 2019). O estudo (E6) (Abhijit et al., 2019), apesar de ter apresentado um tema diferente sobre o trabalho das equipes, também 
se referiu ao tema da comunicação que foi abordado pelos autores dos estudos E1 (Johnson, et al., 2015), E2 (Singh et al., 2015), E4 (Munce et al., 2017) e E7 (Obana et al., 2019).

Em relação às barreiras como fatores que influenciaram os resultados do trabalho em equipe nas tomadas de decisão, os estudos E1 (Johnson et al., 2015), E2 (Singh et al., 2015) e E5 (Plant \& Tyson, 2018) citaram as dificuldades de tempo disponível para alguns membros das equipes inter ou multidisciplinares em participarem de reuniões semanais, que eram os momentos onde se estabeleciam as metas. O estudo E5 (Plant \& Tyson, 2018) também cita a falta de envolvimento de alguns membros da equipe e limitações relacionadas ao AVC do paciente.

Já nos estudos E1 (Johnson et al., 2015) e E4 (Munce et al., 2017), a barreira apontada foi a falta de instalações de reabilitação no hospital para a realização das intervenções de reabilitação pela equipe, nos pacientes com AVC, sendo que o estudo E4 (Munce et al., 2017) também cita a ausência de equipamentos. O estudo E1 (Johnson et al., 2015) também evidencia o conhecimento limitado e desconfiança da equipe sobre os serviços disponíveis na comunidade em geral.

O estudo E2 (Singh et al., 2015) cita como barreira, os atritos e prioridades conflitantes da equipe interdisciplinar para com os pacientes, a amostra pequena e a falta de apoio financeiro para implementação e disseminação de recomendações de diretrizes de melhores práticas.

Já o estudo E3 (Liberati et al., 2016) se refere as abordagens diferentes e às vezes conflitantes de médicos de especialidades diferentes, construção de identidades profissionais específicas de enfermeiros e conflitos entre médicos e enfermeiros de diferentes especialidades sobre a regulação da fronteira médico-enfermagem.

E por fim, o estudo E6 (Abhijit et al., 2019) apresenta como barreira, a falta de consenso multidisciplinar na unidade de terapia intensiva antes da transição dos pacientes graves para as medidas de conforto.

Categoria 2. "Quais os profissionais envolvidos nas esquipes de AVC"

Nesta categoria, os profissionais foram citados em ordem de maior para menor número. O enfermeiro foi o único profissional que apareceu participando de todas as equipes inter ou multidisciplinares. Em seguida, quatro estudos incluíram na equipe, o Fisioterapeuta e o Terapeuta Ocupacional: E1 (Johnson et al., 2015), E2 (Singh et al., 2015), E4 (Munce et al., 2017) e E5 (Plant \& Tyson, 2018); o Neurologista apareceu em quatro também: E2 (Singh et al., 2015), E3 (Liberati et al., 2016), E5 (Plant \& Tyson, 2018) e E8 (Yang et al., 2019). O Fonoaudiólogo foi citado em três artigos: E1 (Johnson et al., 2015), E5 (Plant \& Tyson, 2018) e E7 (Obana et al., 2019) e o Assistente Social em dois: E1 (Johnson et al., 2015), E5 (Plant \& Tyson, 2018). Foram citados apenas uma vez: Dentista (E7 (Obana et al., 2019); Farmacêutico E8 (Yang et al., 2019); Fisiatra E1 (Johnson et al., 2015); Higienistas Orais E7 (Obana et al., 2019), Médico Intensivista E6 (Abhijit et al, 2019), Médicos Residentes em Anestesiologia E6 (Abhijit et al, 2019), Nutricionistas E5 (Plant \& Tyson, 2018) e Radiologista E8 (Yang et al., 2019).

\section{Discussão}

Atualmente, não existe um consenso sobre como o trabalho em equipe e a reabilitação devam ser coordenados de uma maneira otimizada para o atendimento a pessoas com AVC em ambientes hospitalares, pois, esse trabalho ainda está em construção (Kushner \& Strasser, 2020). Os esforços para melhorar a adoção de intervenções baseadas em evidências para resultados ideais para os pacientes em países de baixa e média renda são persistentemente prejudicados por vários tipos de barreiras. No entanto, pouco se sabe sobre as estratégias para lidar com essas barreiras para melhorar o atendimento de qualidade ao AVC (Baatiiema et al., 2020).

Uma das barreiras mais importantes observadas é a falta de informações sobre a forma de funcionamento das equipes de saúde que realizam intervenções em pacientes com AVC, no ambiente hospitalar. Por essa razão, esta pesquisa buscou 
identificar fatores facilitadores e dificultadores do trabalho realizado por equipes inter e multidisciplinares nesses pacientes e detectar quais profissionais de saúde estão envolvidos.

Constatou-se na maioria das pesquisas encontradas, que a colaboração e a comunicação aprimorada no trabalho das EID's e EMD's de AVC podem funcionar como um facilitador para a tomada de decisões em intervenções para pacientes (Johnson et al., 2015; Singh et al., 2015; Munce et al., 2017; Abhijit et al., 2019; Obana et al., 2019). Esses resultados estão de acordo com estudos recentes que indicam que a comunicação e colaboração eficazes da equipe interdisciplinar envolvendo pacientes com AVC, familiares, médicos, enfermeiras, fisioterapeutas, terapeutas ocupacionais, fonoaudiólogos, psicólogos, assistentes sociais e outros, são essenciais para maximizar os resultados da reabilitação em que o trabalho em equipe é um processo central e os resultados clínicos obtidos pelos pacientes, são essenciais para avaliar a qualidade dos cuidados pósagudos (Dinius et al., 2020).

Também, foram encontrados temas relacionados ao trabalho de equipes inter e multidisciplinares, mas de forma bastante diversificada. Os diferentes temas apresentados nesses estudos foram: a regulação do trabalho sob a fronteira médicoenfermagem (Liberati et al., 2016), os terapeutas sendo maioria na identificação de deficiências dos pacientes com AVC (Plant \& Tyson, 2018), a implementação de consenso de medidas de conforto para esses pacientes (Abhijit et al, 2019) e a melhora no tempo de reconhecimento do início dos sintomas de ocorrência de AVC em pacientes internados (Yang et al., 2019). Á partir dessa configuração, notou-se a grande preocupação por parte desses autores, em estudar os aspectos facilitadores do trabalho em equipe, porque esses aspectos estavam ligados as particularidades de cada tipo de serviço prestado a pacientes com AVC no ambiente hospitalar.

Essa variedade de temas pode ser relacionada, primeiro, ao fato de pessoas com AVC e profissionais apresentarem características diferentes, que faz parte da natureza humana; e, segundo, à importância e à necessidade de individualização do cuidado prestado, pois, os pacientes com AVC têm sequelas e necessidades bastante diferentes, fator que justifica a mudança na atuação profissional de um lugar para o outro. Uma explicação apontada para a diversidade de temas e profissionais envolvidos no trabalho das equipes multidisciplinares, se dá por conta do progresso da medicina estar relacionado à crescente especialização das profissões médicas e isso levar à fragmentação do trabalho. Somando-se a esse aspecto, acrescenta-se o fato do envelhecimento da população conduzir ao aumento de doenças crônicas, o que torna o atendimento ao paciente cada vez mais complexo (Kilpatrick et al., 2020).

Quanto às barreiras para o trabalho inter ou multidisciplinar, evidenciamos como principais, a falta de tempo (Johnson et al., 2015; Singh et al., 2015; Plant \& Tyson, 2018) e falta de envolvimento de membros da equipe (Plant \& Tyson et al., 2018) para esse tipo de intervenção. Esses fatores afetam o estabelecimento de metas para a tomada de decisão da equipe e isso se torna ainda mais importante, ao verificar em estudos recentes que o próprio funcionamento da equipe também é influenciado por processos que incluem a tomada de decisão (Voogdt-Pruis, 2019; Kilpatrick et al., 2020).

Outros fatores dificultadores que também afetam o estabelecimento de metas da equipe, são: a diversidade que existe na composição interna do corpo de profissionais, o que torna o foco, tipo de trabalho e tipo de tomada de decisão, diferentes. No entanto, isso se torna compreensível e justificável frente às diferentes sequelas ou necessidades comuns que demandam cuidados entre os pacientes que sofreram AVC (Schiavi, 2018).

Dessa forma e apesar da escassez de literatura acerca do tema sobre como funciona o trabalho das equipes inter e multidisciplinares, é notória a possibilidade de realização do trabalho em equipe no atendimento a vítimas de AVC. Também é evidente a importância do entendimento das possíveis formas de trabalho desses grupos e quem são os profissionais envolvidos, pois, as equipes são sistemas ativos de aprendizagem, nos quais os indivíduos desenvolvem relacionamentos e aplicam o conhecimento para resolver problemas relacionados aos pacientes internados, entre eles, os que são acometidos por um AVC (Schiavi et al., 2018; Amatangelo et al., 2020; Schwarzbach et al., 2020; Swank, 2020). 


\section{Limitações do Estudo}

Embora tenhamos seguido uma abordagem padronizada para a pesquisa da literatura, reconhece-se as limitações. Por exemplo, diferenças nas abordagens metodológicas e além disso, ainda que a pesquisa tenha ocorrido em nove bases de dados, o resultado de outras pesquisas que poderiam estar disponíveis, não foi acessado.

\section{Conclusão}

As pesquisas apresentadas nessa revisão evidenciaram que os profissionais envolvidos no trabalho interdisciplinar e multidisciplinar com pacientes de AVC são diversos e apesar de enfermeiros, fisioterapeutas e médicos com formação específica serem fundamentais no tratamento desses pacientes, o enfermeiro foi o único profissional encontrado em todos os estudos.

Além disso, observou-se que os fatores facilitadores e dificultadores do trabalho da equipe relacionaram-se ao tipo de necessidade de cuidado desses pacientes, principalmente por conta do trabalho para a população de AVC, costumeiramente ser bastante diversificado, devido a múltiplas e diferentes sequelas que são apresentadas.

Dada a escassez de estudos que relatem a forma de funcionamento das equipes interdisciplinares e multidisciplinares, recomenda-se que novas pesquisas sejam realizadas.

Compreender como os membros dessas equipes com diferentes experiências profissionais atuam juntos, é crucial para avaliar plenamente o contexto do paciente com doenças crônicas, como o AVC, e trabalhar em direção ao planejamento holístico do cuidado.

Sugere-se assim, aprofundar os estudos acerca dessa temática, com o objetivo de identificar e elaborar estratégias de trabalho em equipes que possam garantir o cuidado adequado de pessoas com AVC em ambientes hospitalares, respeitando-se as particularidades dessa doença, a sutileza das relações humanas, as especificidades de cada profissão e valorizando a inter e multidisciplinaridade.

\section{Agradecimentos}

Agradecemos a pesquisadora Enfa. Ms. Mariana Véo Nery de Jesus (in memorian) pela participação nesse trabalho como a terceira revisora e ao Prof. Dr. Ricardo José Orsi de Sanctis pela revisão gramatical da língua espanhola.

\section{Referências}

Abhijit, L., Cheever, C., Healey, L., Hurley, K., Kim, L. \& Creutzfeldt, C. (2019). Operationalization of the Transition to Comfort Measures Only in the Neurocritical Care Unit: A Quality Improvement Project. American Journal of Hospice \& Palliative Medicine. 36(1):38-44. https://doi.org/10.1177/1049909118790069.

Amatangelo, M. \& Thomas, S. (2020). Priority Nursing Interventions Caring for the Stroke Patient. Critical Care Nursing Clinics of North America. 32(1):6784. https://doi.org/10.1016/j.cnc.2019.11.005.

Baatiema, L., Aikins, A., Sarfo, F., Abimbola, S., Ganle, J. \& Somerset, S. (2020). Improving the quality of care for people who had a stroke in alow-/middleincome country: A qualitative analysis of healthcare professionals' perspectives. Health Expectations. 00:1-11. https://doi: 10.1111/hex.13027.

Becker. S., Korner, M., Muller, C., Lippenberger, C., Rundel, M. \& Zimmermann, L. (2017). Development and pilot testing of an interprofessional patientcentered team training programme in medical rehabilitation clinics in Germany: a process evaluation. Medical Education. 17:120, 1-9. https://doi: 10.1186/s12909-017-0960.

Bjerkreim, A., Thomassen, L., Waje_Andreassen, U., Selvik, H. \& Naess, H. (2016). Hospital Readmission after Intracerebral Hemorrhage. Journal of Stroke and Cerebrovascular Diseases. 25(1), 157-62. https://doi: 10.1016/j.jstrokecerebrovasdis.

Coupland, A. P., Thapar, A., Qureshi, M.I., Jenkins, H. \& Davies A.H. (2017). The definition of stroke. Journal of the Royal Society of Medicine. 110(1), 9-12. https://doi/full/10.1177/0141076816680121.

Dinius, J., Philipp, R., Ernstmann, N., Heier, L., Goritz, A., Pfisterer_Heise, S., Hammerschmidt, J., Bergelt, C., Hammer, A. \& Korner, M. (2020). Interprofessional teamwork and its association with patient safety in German hospitals-A cross sectional study. Plos One. 15(5):1-15. https://doi.org/10.1371/journal.pone.0233766. 
Feigin, V.L., Nichols, E., Alam, T., Bannick, M.S., Beghi, E., Blake, N. \& Vos, T. (2019). Global, regional, and national burden of neurological disorders, 1990-2016: A systematic analysis for the Global Burden of Disease Study 2016. The Lancet Neurology, 18(5), 459-480. https://doi:10.1016/S14744422(18)30499-X.

Feigin, V.L., Norrving, B. \& Mensah, G.A. (2017). Global burden of stroke. Circulation Research. 120 (3), 439-448. https://doi: 10.1055/s-0038-1649503.

Hustoft, M., Biringer, E., Gjesdal, S., Abmus, J. \& Hetlevik, O. (2018) Relational coordination in interprofessional teams and its effect on patient-reported benefit and continuity of care: a prospective cohort study from rehabilitation centres in Western Norway. BMC Health Services Research. 18:719, 1-9. https://doi: 10.1186/s12913-018-3536-5.

Intercollegiate Stroke Working Party. (2017). National Clinical Guidelines for Stroke. 4th ed. London: Royal College of Physicians. https://www.strokeaudit.org/SupportFiles/Documents/Guidelines/2016-National-Clinical-Guideline-for-Stroke-5t-(1).aspx.

Johnson, J., Smith, G. \& Wilkinson, A. (2015). Factors that influence the decision-making of an interdisciplinary rehabilitation team when choosing a discharge destination for stroke survivors. Canadian Journal of Neuroscience Nursing. 37(2):26-32. https://europepmc.org/article/med/26647491.

Kilpatrick, K., Paquette, L., Jabbour, M., Tchouaket, E., Fernandez, N., Hakim, G., Landry, V., Gauthier, N., Beaulieu, M.D. \& Dubois, C. (2020). Systematic review of the characteristics of brief team interventions to clarify roles and improve functioning in healthcare teams. PloS One. $15(6)$ : 0234416. https://doi.org/10.1371/ journal.pone.0234416.

Koton, S. \& Rexrode, K.M. (2017). Trends in stroke incidence in the United States. Neurology. 89 (10): 982. https://doi:10.1212/wnl.0000000000004342.

Kushner, D. \& Strasser, D. (2020). Stroke Inpatient Rehabilitation Team Conferences: Leadership and Structure Improve Patient Outcomes. Journal of Stroke and Cerebrovascular Diseases. 29(4):1-9. https://doi.org/10.1016/j.jstrokecerebrovasdis.2019.104622.

Liberati, E.G., Gorli, M. \& Scaratti, G. (2016). Invisible walls within multidisciplinary teams: Disciplinary boundaries and their effects on integrated care. Social Science \& Medicine. 150:31-9. https://doi:10.1016/j.socscimed.2015.12.002.

Mendes K.D.S., Silveira R.C.C.P. \& Galvão, C.M. (2008). Revisão integrativa da literatura: um método de pesquisa para incorporar evidências em saúde e enfermagem. Texto e Contexto Enfermagem. 17 (4): 758-64. https://doi.org/10.1590/S0104-07072008000400018.

Mother, D., Liberati, A., Tetzlaff, J. \& Altman, D.G. (2009). The PRISMA Group. Preferred Reporting Items for Sistematic Reviews and Meta-Analyses: The PRISMA Statement for reporting systematic reviews and meta-analyses of studies that evaluate health care interventions: explanation and elaboration. Journal of Clinical Epidemiology. 62(10):1006-12. https://doi:10.1371/journal.pmed.1000100.

Munce S.E.P., Graham, I.D. \& Salbach N.M. (2017). Perspectives of health care professionals on the facilitators and barriers to the implementation of a stroke rehabilitation guidelines cluster randomized controlled trial. BMC Health Services Research. 17(1):440. https://doi:10.1186/s12913-017-2389-7.

Ngangue, P., Forques, C., Nguyen, T., Sasseville, M., Gallagher, F., Loignon, C., Stewart, M., Belle, B., Chouinard, M.C. \& Fortin, M. (2020). Patients, caregivers and health-care professionals' experience with an interdisciplinary intervention for people with multimorbidity in primary care: A qualitative study. Health Expectations. 23. 318-327. https://doi: 10.1111/hex.13035.

Obana, M., Furuya, I., Matsubara, C., Tohara, H., Inaji, M., Miki, K., Numasawa, Y. \& Minakuchi, S. (2019). Effect of a collaborative transdisciplinary team approach on oral health status in acute stroke patients. Journal of Oral Rehabilitation. 46:1170-1176. https://doi.org/10.1111/joor.12855.

Philip B.G. (2019). The global burden of stroke: persistent and disabling. Lancet. 18, 417, March 11. https://doi: 10.1016/ S1474-4422(19)30030-4.

Plant, S. \& Tyson S.F. (2018). A multicentre study of how goal-setting is practised during inpatient stroke rehabilitation. Clinical Rehabilitation. 32(2):263272. https://doi: 10.1177/0269215517719485.

Ranford, J., Asiello, J., Cloutier, A., Cortina,K., Thorne, H., Erler, K., Frazier, N., Sadlak, C., Rude, A. \& Lin, D. (2019). Interdisciplinary Stroke Recovery Research: The perspective of Occupational Therapists in Acute Care. Frontiers in Neurology. V. 10, p.1-6. https://doi: 10.3389/fneur.2019.01327.

Schiavi, M., Costi, S., Pellegrini, M., Formisano, D., Borghi, S. \& Fugazzaro, S. (2018). Occupational therapy for complex inpatients with stroke: identification of occupational needs in post-acute rehabilitation setting. Disability and rehabilitation. 40(9):1026-1032. https://doi.org/10.1080/09638288.2017.1283449.

Schwarzbach, C. \& Grau, A.J. (2020). Complications after stroke: Clinical challenges in stroke aftercare. Der Nervenarzt. 91(10): 920-925. https://doi.org/ 10.1007/s00115-020-00988-9.

Singh, M., Hynie, M., Rivera, T., Macisaac, L., Gladman, A., \& Cheng, A. (2015). An evaluation study of the implementation of stroke best practice guidelines using a Knowledge Transfer Team approach. Canadian Journal of Neuroscience Nursing. 37(1):24-33. https://pubmed.ncbi.nlm.nih.gov/26152100/.

Singh, R., Küçükdeveci, A., Grabljevec, K. \& Gray, A. (2018). The Role of Interdisciplinary Teams in Physical and Rehabilitation Medicine. Journal of Rehabilitation Medicine. V.50. P. 673-678. https://doi: 10.2340/16501977-2364.

Stillwel, S.B., Fineout-Overholt, E., Melnyk, B.M. \& Williamson, K.M. (2010). Evidence-based practice, step by step: searching for the evidence. American Journal of Nursing. 110(5):41-7. https://doi: 10.1097/01.naj.0000372071.24134.7e.

Swank, C. (2020). The impact of a patient-directed activity program on functional outcomes and activity participation after stroke during inpatient rehabilitation-a randomized controlled trial. Clinical Rehabilitation. 34(4):504-514. https://doi.org/10.1177/0269215519901153.

Ursi, E.S. \& Galvão, C.M. (2006). Prevenção perioperatória de lesão de pele: revisão integrativa da literatura. Revista Latino-Americana de Enfermagem. jan / fev; 14 (1): 124-31. https://doi.org/10.1590/S0104-11692006000100017. 
Research, Society and Development, v. 10, n. 9, e39010918012, 2021

(CC BY 4.0) | ISSN 2525-3409 | DOI: http://dx.doi.org/10.33448/rsd-v10i9.18012

Voogdt_Pruis, H., Ras, T., Van der Dussen, L., Benjaminsen, S., Goossens, P.H., Raats, I., Boss, G., Van Hoef, E.F.M., Lindhout, M., Tjon-A-Tsien, M.R.S. \& Vrijhoef, H.J.M. (2019). Improvement of shared decision making in integrated stroke care: a before and after evaluation using a questionnaire survey. BMC Health Services Research. 19(936):1-22. https://doi.org/10.1186/s12913-019-4761-2.

Xiamei, M., Xuemei, C., Zhihui, L. \& Lanshu, Z. (2019). Nursing practice in stroke rehabilitation: Perspectives from multi-disciplinary healthcare professionals. Nursing \& Health Sciences. 1-10. https://doi: 10.1111/nhs.12641.

Walton, V., Hogden, A., Long, J., Johnson, J. \& Greenfield, D. (2019). How Do Interprofessional Healthcare Teams Perceive the Benefits and Challenges of Interdisciplinary Ward Rounds. Journal of Multidisciplinary Healthcare. V. 12. P. 1023-1032. https://doi: 10.2147/JMDH.S226330.

Yang, S., Franco, T., Wallace, N., Williams, B. \& Blackmore, C. (2019). Effectiveness of an Interdisciplinary, Nurse Driven In-Hospital Code Stroke Protocol on In-Patient Ischemic Stroke Recognition and Management. Journal of Stroke and Cerebrovascular Diseases, 28, (12):104398. https://doi: org/10.1016/j.jstrokecerebrovasdis.2019.104398. 